\title{
Inference on Exponentiated Power Lindley Distribution Based on Order Statistics with Application
}

\author{
Mansour Shrahili $\mathbb{D}^{1},{ }^{1}$ Naif Alotaibi $\mathbb{D}^{2},{ }^{2}$ Devendra Kumar ${ }^{(D)},{ }^{3}$ and A. R. Shafay ${ }^{4,5}$ \\ ${ }^{1}$ Department of Statistics and Operations Research, King Saud University, Riyadh, Saudi Arabia \\ ${ }^{2}$ Department of Mathematics and Statistics, Imam Mohammad Ibn Saud Islamic University, Riyadh, Saudi Arabia \\ ${ }^{3}$ Department of Statistics, Central University of Haryana, Mahendergarh, India \\ ${ }^{4}$ Natural Science Department, Community College of Riyadh, King Saud University, Riyadh, Saudi Arabia \\ ${ }^{5}$ Department of Mathematics, Faculty of Science, Fayoum University, Fayoum, Egypt \\ Correspondence should be addressed to Mansour Shrahili; msharahili@ksu.edu.sa
}

Received 12 April 2020; Revised 15 July 2020; Accepted 16 July 2020; Published 23 September 2020

Academic Editor: Michele Scarpiniti

Copyright (c) 2020 Mansour Shrahili et al. This is an open access article distributed under the Creative Commons Attribution License, which permits unrestricted use, distribution, and reproduction in any medium, provided the original work is properly cited.

\begin{abstract}
Exponentiated power Lindley distribution is proposed as a generalization of some widely well-known distributions such as Lindley, power Lindley, and generalized Lindley distributions. In this paper, the exact explicit expressions for moments of order statistics from the exponentiated power Lindley distribution are derived. By using these relations, the best linear unbiased estimates of the location and scale parameters, based on type-II right-censored sample, are obtained. Next, the mean, variance, and coefficients of skewness and kurtosis of some certain linear functions of order statistics are calculated and then used to derive the approximate confidence interval for the location and scale parameters using the Edgeworth approximation. Finally, some numerical illustrations and two real data applications are presented.
\end{abstract}

\section{Introduction}

Ashour and Eltehiwy [1] introduced the exponentiated power Lindley (EPL) distribution as a generalization of twoparameter power Lindley distribution and they studied some mathematical properties of the EPL distribution. They also showed that this distribution provides more flexibility to analyze a complex real data set. Due to its practicality, the EPL distribution can be used for many applications, including accelerated life testing, survival analysis, reliability, biology, and others. The EPL distribution has attractive feature that its hazard rate function could be decreasing, increasing, and decreasing-increasing-decreasing but not constant, depending on the shape parameter. The EPL distribution has a unimodal and right skewed probability density function (PDF) for $\delta>1$. Many authors have developed generalization of Lindley distribution. Prominent among these generalizations which we are aware of are generalized Lindley distribution by Nadarajah et al. [2]; power Lindley distribution by Ghitany et al. [3]; generalized inverse Lindley distribution by Sharma et al. [4]; pseudoLindley distribution by Nedjar and Zeghdoudi [5]; sizebiased gamma Lindley distribution by Beghriche and Zeghdoud [6]. Ashour and Eltehiwy [1] observed that in numerous situations, the EPL distribution provided a better fit than Lindley, power Lindley, generalized Lindley, exponentiated exponential, modified Weibull, and Weibull distributions.

Over the past six decades or so, several authors have shown keen interest in order statistics. These order statistics and their moments are of great significance in many real life applications involving data relating to flood, drought, reliability, engineering, and life testing situation. In recent times, several papers and books have been published on order statistics and their distributional properties. Among them are David and Nagaraja [7]; Arnold et al. [8]; Balakrishnan and Cohen [9]; Balakrishnan and Ahsanullah [10]; Balakrishnan and Sultan [11]; Malik et al. [12]; 
Mahmoud et al. [13]; Genc [14]; MirMostafaee [15]; Balakrishnan et al. [16]; Kumar and Dey [17]; Sultan et al. [18]; Sultan and AL-Thubyani [19]; Kumar et al. [20]; and so on. Due to the wide applications of order statistics, several authors have carried out extensive studies on these kinds of ordered data. Numerous papers dealing with moments and estimation of parameters of different lifetime distributions based on order statistics can be found in literature. For example, Balakrishnan and Cohen [9] discussed the moments and estimation of parameters. Sultan et al. [18] obtained the moments of power function distribution. Sultan and AL-Thubyani [19] developed the higher-order moments and inferential procedure for estimating parameters of Lindley distribution. Ahsanullah and Alzaatreh [21] studied the moments and inferential procedure for log-logistic distribution. Recently, Kumar and Goyal [22, 23] and Kumar et al. [24, 25] developed inferential procedure for power Lindley and generalized Lindley, modified power function, and generalized inverse Lindley distribution.

Let $Y_{1}, Y_{2}, \ldots, Y_{m}$ be an independent random variable from the EPL distribution with cumulative distribution function (CDF) and PDF is given, respectively, by

$$
F(y)=\left[1-\left(\frac{1+v+v y^{\gamma}}{1+v}\right) \exp \left(-v y^{\gamma}\right)\right]^{\delta}, \quad y>0, \delta>0, v>0, \gamma>0
$$

$$
\begin{aligned}
f(y)= & \frac{\delta v^{2} \gamma y^{\gamma-1}}{1+v}\left(1+y^{\gamma}\right) \exp \left(-v y^{\gamma}\right) \\
& \cdot\left[1-\left(\frac{1+v+v y^{\gamma}}{1+v}\right) \exp \left(-v y^{\gamma}\right)\right]^{\delta-1}, \quad y>0 .
\end{aligned}
$$

The hazard function of the EPL distribution is given by

$$
\begin{aligned}
h(y)= & \frac{\delta v^{2} \gamma y^{\gamma-1}}{1+v}\left(1+y^{\gamma}\right)[V(y)]^{\delta-1} \\
& \cdot\left[1-V^{\delta}(y)\right]^{-1} \exp \left(-v y^{\gamma}\right), \quad y>0, \delta, v, \gamma>0,
\end{aligned}
$$

where

$$
V(y)=\left[1-\left(\frac{1+v+v y}{1+v}\right) \exp \left(-v y^{\gamma}\right)\right]
$$

Some widely well-known distributions can be obtained as special cases from the EPL distribution. By setting $\gamma=1$ and $\delta=1$ in both (1) and (2), the CDF and PDF of the generalized Lindley distribution and power Lindley distribution are obtained, respectively, while setting $\delta=\gamma=1$ gives the CDF and PDF of Lindley distributions.

To the best of our knowledge, there are no reports on moments and estimation for the EPL distribution using order statistics. In this paper, first is to obtain the exact explicit expressions for moments of order statistics from the EPL distribution, and second is to derive the best linear unbiased estimates (BLUEs) of the location and scale parameters of the EPL distribution based on order statistics.

The rest of this paper is organized as follows: in Section 2, the moments of EPL distribution are derived based on order statistics. The BLUEs of the location and scale parameters are provided in Section 3. Next, these BLUEs are used in Section 4 to obtain the coefficient of skewness and kurtosis of some pivotal quantities. Also, we obtain Edgeworth approximations for the distributions of these pivotal quantities. Simulation study and real data analysis are presented in Sections 5 and 6. Finally, the paper ends with a conclusion in Section 7.

\section{Moments of Order Statistics}

In this section, the moments of order statistics are established for a given random sample $Y_{1}, Y_{2}, \ldots, Y_{m}$ from the EPL distribution.

2.1. Single Moments. In this subsection, the explicit expressions for single moments of order statistics are derived. Let $Y_{1: m} \leq Y_{2: m} \leq \cdots \leq Y_{m: m}$ be the order statistics for a given random sample $Y_{1}, Y_{2}, \ldots, Y_{m}$ from the EPL distribution, and $Y_{r: m}, r=1,2, \ldots, m$, denotes the $r$ th order statistics with PDF which is given by (see [7])

$\% f_{Y_{r: m}}(x)=C_{r: m} F^{r-1}(y)[1-F(y)]^{m-r} f(y), \quad-\infty<y<\infty$,

where $F($.$) and f($.$) are given by (1) and (2), respectively,$ and

$$
C_{r: m}=\frac{m !}{(r-1) !(m-r) !} .
$$

Theorem 1. For the EPL distribution given in (1), the $p_{1}$ th moment of the rth order statistics $\mu_{r: m}^{\left(P_{1}\right)}, p_{1}=0,1, \ldots$ and $1 \leq r \leq m$, is as follows:

$$
\begin{aligned}
\mu_{r: m}^{\left(p_{1}\right)}= & C_{r: m} \frac{\delta v^{2}}{(1+v)} \sum_{j_{1}=0}^{m-r} \sum_{j_{2}=0}^{\delta\left(r+j_{1}\right)-1} \sum_{j_{3}=0}^{j_{2}} \sum_{j_{4}=0}^{j_{3}+1}(-1)^{j_{1}+j_{2}} \frac{v^{j_{3}}}{(1+v)^{j_{2}}}\left(\begin{array}{c}
m-r \\
j_{1}
\end{array}\right) \\
& \times\left(\begin{array}{c}
\delta\left(r+j_{1}\right)-1 \\
j_{2}
\end{array}\right)\left(\begin{array}{c}
j_{2} \\
j_{3}
\end{array}\right)\left(\begin{array}{c}
j_{3}+1 \\
j_{4}
\end{array}\right) \frac{\Gamma\left(\left(p_{1}+\gamma\left(j_{4}+1\right)\right) / \gamma\right)}{\left[v\left(j_{2}+1\right)\right]^{\left(p_{1}+\gamma\left(j_{4}+1\right)\right) / \gamma} .} .
\end{aligned}
$$


Proof. From (5), we have

$$
\begin{aligned}
\mu_{r: m}^{\left(p_{1}\right)} & =E\left(Y_{r: m}^{\left(p_{1}\right)}\right)=C_{r: m} \int_{0}^{\infty} y^{p_{1}} F^{r-1}(y)[1-F(y)]^{m-r} f(y) \\
& =C_{r: m} \sum_{j_{1}=0}^{m-r}(-1)^{j_{1}}\left(\begin{array}{c}
m-r \\
j_{1}
\end{array}\right) \int_{0}^{\infty} y^{p_{1}}[F(y)]^{j_{1}+r-1} f(y) \mathrm{d} y .
\end{aligned}
$$

By using (1) and (2), one can write

$$
\begin{aligned}
\mu_{r: m}^{\left(p_{1}\right)}= & C_{r: m} \frac{\delta \gamma v^{2}}{(1+v)} \sum_{j_{1}=0}^{m-r}(-1)^{j_{1}}\left(\begin{array}{c}
m-r \\
j_{1}
\end{array}\right) \\
& \cdot \int_{0}^{\infty} y^{p_{1}+\gamma-1}\left(1+y^{\gamma}\right) e^{-v y^{\gamma}} \\
& \times\left[1-\left(\frac{1+v+v y^{\gamma}}{1+v}\right) e^{-v y^{\gamma}}\right]^{\delta\left(r+j_{1}\right)-1} \mathrm{~d} y \\
= & C_{r: m} \frac{\delta \gamma v^{2}}{(1+v)} \sum_{j_{1}=0}^{m-r} \delta\left(\sum_{j_{2}=0}^{\left.r+j_{1}\right)-1} \sum_{j_{3}=0}^{j_{2}} \sum_{j_{4}=0}^{j_{3}+1}(-1)^{j_{1}+j_{2}}\right. \\
& \cdot \frac{v^{j_{3}}}{(1+v)^{j_{2}}}\left(\begin{array}{c}
m-r \\
j_{1}
\end{array}\right)\left(\begin{array}{c}
\delta\left(r+j_{1}\right)-1 \\
j_{2}
\end{array}\right) \\
& \times\left(\begin{array}{l}
j_{3}+1 \\
\left.j_{3}\right)\left(j_{4} y_{0}^{p_{1}+\gamma\left(j_{4}+1\right)-1} e^{-v\left(j_{2}+1\right) y^{\gamma}} \mathrm{d} y\right.
\end{array}\right.
\end{aligned}
$$

hence the result.

Some special cases from equation (7):

(1) If $\delta=1$, we get

$$
\begin{aligned}
\mu_{r: m}^{\left(p_{1}\right)}= & C_{r: m} \frac{v^{2}}{(1+v)} \sum_{j_{1}=0}^{m-r} \sum_{j_{2}=0}^{r+j_{1}-1} \sum_{j_{3}=0}^{j_{2}} \sum_{j_{4}=0}^{j_{3}+1}(-1)^{j_{1}+j_{2}} \frac{v^{j_{3}}}{(1+v)^{j_{2}}}\left(\begin{array}{c}
m-r \\
j_{1}
\end{array}\right) \\
& \times\left(\begin{array}{c}
r+j_{1}-1 \\
j_{2}
\end{array}\right)\left(\begin{array}{c}
j_{2} \\
j_{3}
\end{array}\right)\left(\begin{array}{c}
j_{3}+1 \\
j_{4}
\end{array}\right) \frac{\Gamma\left(\left(p_{1}+\gamma\left(j_{4}+1\right)\right) / \gamma\right)}{\left[v\left(j_{2}+1\right)\right]^{\left(p_{1}+\gamma\left(j_{4}+1\right)\right) / \gamma}} .
\end{aligned}
$$

(2) If $\gamma=1$, we get

$$
\begin{aligned}
\mu_{r: m}^{\left(p_{1}\right)}= & C_{r: m} \frac{\delta v^{2}}{(1+v)} \sum_{j_{1}=0}^{m-r} \sum_{j_{2}=0}^{\delta\left(r+j_{1}\right)-1} \sum_{j_{3}=0}^{j_{2}} \sum_{j_{4}=0}^{j_{3}+1}(-1)^{j_{1}+j_{2}} \frac{v^{j_{3}}}{(1+v)^{j_{2}}}\left(\begin{array}{c}
m-r \\
j_{1}
\end{array}\right) \\
& \times\left(\begin{array}{c}
\delta\left(r+j_{1}\right)-1 \\
j_{2}
\end{array}\right)\left(\begin{array}{c}
j_{2} \\
j_{3}
\end{array}\right)\left(\begin{array}{c}
j_{3}+1 \\
j_{4}
\end{array}\right) \frac{\Gamma\left(p_{1}+j_{4}+1\right)}{\left[v\left(j_{2}+1\right)\right]^{p_{1}+j_{4}+1}},
\end{aligned}
$$

as obtained by Kumar and Goyal [23].

(3) If $\gamma=1$ and $\delta=1$, we get

$$
\begin{aligned}
\mu_{r: m}^{\left(p_{1}\right)}= & C_{r: m} \frac{v^{2}}{(1+v)} \sum_{j_{1}=0}^{m-r} \sum_{j_{2}=0}^{r+j_{1}-1} \sum_{j_{3}=0}^{j_{2}} \sum_{j_{4}=0}^{j_{3}+1}(-1)^{j_{1}+j_{2}} \frac{v^{j_{3}}}{(1+v)^{j_{2}}}\left(\begin{array}{c}
m-r \\
j_{1}
\end{array}\right) \\
& \times\left(\begin{array}{c}
r+j_{1}-1 \\
j_{2}
\end{array}\right)\left(\begin{array}{c}
j_{2} \\
j_{3}
\end{array}\right)\left(\begin{array}{c}
j_{3}+1 \\
j_{4}
\end{array}\right) \frac{\Gamma\left(p_{1}+j_{4}+1\right)}{\left[v\left(j_{2}+1\right)\right]^{p_{1}+j_{4}+1}} .
\end{aligned}
$$

(4) If $p_{1}=1$ and $r=1$, we get

$$
\begin{aligned}
\mu_{1: m}^{(1)}= & \frac{m \delta v^{2}}{(1+v)} \sum_{j_{1}=0}^{m-1} \sum_{j_{2}=0}^{\delta\left(r+j_{1}\right)-1} \sum_{j_{3}=0}^{j_{2}} \sum_{j_{4}=0}^{j_{3}+1}(-1)^{j_{1}+j_{2}} \frac{v^{j_{3}}}{(1+v)^{j_{2}}}\left(\begin{array}{c}
m-1 \\
j_{1}
\end{array}\right) \\
& \times\left(\begin{array}{c}
\delta\left(j_{1}+1\right)-1 \\
j_{2}
\end{array}\right)\left(\begin{array}{c}
j_{2} \\
j_{3}
\end{array}\right)\left(\begin{array}{c}
j_{3}+1 \\
j_{4}
\end{array}\right) \frac{\Gamma\left(\left(1+\gamma\left(j_{4}+1\right)\right) / \gamma\right)}{\left[v\left(j_{2}+1\right)\right]^{\left(1+\gamma\left(j_{4}+1\right)\right) / \gamma}} .
\end{aligned}
$$

(5) If $p_{1}=1$ and $r=m$, we get

$$
\begin{aligned}
\mu_{m: m}^{(1)}= & \frac{m \delta v^{2}}{(1+v)} \sum_{j_{2}=0}^{\delta m-1} \sum_{j_{3}=0}^{j_{2}} \sum_{j_{4}=0}^{j_{3}+1}(-1)^{j_{2}} \frac{v^{j_{3}}}{(1+v)^{j_{2}}} \\
& \times\left(\begin{array}{c}
\delta m-1 \\
j_{2}
\end{array}\right)\left(\begin{array}{c}
j_{2} \\
j_{3}
\end{array}\right)\left(\begin{array}{c}
j_{3}+1 \\
j_{4}
\end{array}\right) \frac{\Gamma\left(\left(1+\gamma\left(j_{4}+1\right)\right) / \gamma\right)}{\left[v\left(j_{2}+1\right)\right]^{\left(1+\gamma\left(j_{4}+1\right)\right) / \gamma}} .
\end{aligned}
$$

(6) If $p_{1}=1$ and $m=r=1$, we get 


$$
\begin{aligned}
\mu_{1: 1}^{(1)}= & \frac{\delta v^{2}}{(1+v)} \sum_{j_{2}=0}^{\delta-1} \sum_{j_{3}=0}^{j_{2}} \sum_{j_{4}=0}^{j_{3}+1}(-1)^{j_{2}} \frac{v^{j_{3}}}{(1+v)^{j_{2}}} \\
& \cdot\left(\begin{array}{c}
\delta-1 \\
j_{2}
\end{array}\right)\left(\begin{array}{c}
j_{2} \\
j_{3}
\end{array}\right)\left(\begin{array}{c}
j_{3}+1 \\
j_{4}
\end{array}\right) \frac{\Gamma\left(\left(\gamma\left(j_{4}+1\right)+1\right) / \gamma\right)}{\left[v\left(j_{2}+1\right)\right]^{\left(\gamma\left(j_{4}+1\right)+1\right) / \gamma}} \\
= & E(Y),
\end{aligned}
$$

as obtained by Ashour and Eltehiwy [1].
2.2. Double Moments. Let $Y_{1: m} \leq Y_{2: m} \leq \cdots \leq Y_{n: m}$ be the order statistics from the EPL distribution. Then, the joint PDF of the $r$ th and sth order statistics is given as

$$
\begin{aligned}
f_{Y_{(r: m)}, Y_{(s: m)}}(y, z)= & C_{r, s: m} F^{r-1}(y)[F(z)-F(y)]^{s-1-r} \\
& \cdot[1-F(z)]^{m-s} f(y) f(z), \quad 0<y<z,
\end{aligned}
$$

where $\quad 1 \leq r<s \leq m \quad$ and $C_{r, s: m}=(m ! /((r-1) !(s-r-1) !(m-s) !))$.

Theorem 2. For the EPL distribution given in (1), the double moment of $r$ th and sth order statistics, $\mu_{r, s:}^{\left(p_{1}, p_{2}\right)}$, $p_{1}, p_{2}=1,2, \ldots$, is as follows:

$$
\begin{aligned}
\mu_{r, s: m}^{\left(p_{1}, p_{2}\right)=}= & C_{r, s: m} \frac{\delta^{2} v^{4}}{(1+v)} \sum_{j_{1}=0}^{s-r-1} \sum_{j_{2}=0}^{m-s} \sum_{j_{3}=0}^{\delta\left(r+j_{1}\right)-1} \sum_{i_{4}=0}^{\delta\left(s-r+j_{2}-j_{1}\right)-1} \sum_{j_{5}=0}^{j_{3}} \sum_{i_{6}=0}^{j_{4}} \sum_{i_{7}=0}^{j_{5}+1} \sum_{j_{8}=0}^{j_{6}+1} \sum_{j_{9}=0}^{\left(\left(p_{1}+\gamma\left(j_{8}+1\right)\right) / \gamma\right)-1} \\
& \times(-1)^{j_{1}+j_{2}+j_{3}+j_{4}} \frac{v^{j_{5}+j_{6}}}{(1+v)^{j_{1}+j_{3}+j_{4}}}\left(\begin{array}{c}
s-r-1 \\
j_{1}
\end{array}\right)\left(\begin{array}{c}
m-s \\
j_{2}
\end{array}\right)\left(\begin{array}{c}
\delta\left(r+j_{1}\right)-1 \\
j_{3}
\end{array}\right)\left(\begin{array}{c}
j_{3} \\
j_{5}
\end{array}\right)\left(\begin{array}{c}
j_{4} \\
j_{6}
\end{array}\right)\left(\begin{array}{c}
j_{5}+1 \\
j_{7}
\end{array}\right)\left(\begin{array}{c}
j_{6}+1 \\
j_{8}
\end{array}\right) \\
& \times\left(\begin{array}{c}
\delta\left(s-r+j_{2}-j_{1}\right)-1 \\
j_{4}
\end{array}\right) \\
& \times \frac{\left[v\left(j_{4}+1\right)\right]^{j_{9}-\left(\left(p_{1}+\gamma\left(j_{8}+1\right)\right) / \gamma\right)}\left(\left(\left(p_{1}+\gamma\left(j_{8}+1\right)\right) / \gamma\right)-1\right) !}{j_{9} !} \frac{\Gamma\left(\left(p_{1}+\gamma\left(j_{8}+1\right) / \gamma\right)+1\right)}{\left[v\left(j_{3}+j_{4}+2\right)\right]} .
\end{aligned}
$$

Proof. Using (16), we have

$$
\mu_{r, s: m}^{\left(p_{1}, p_{2}\right)}=E\left(Y_{r, s: m}^{\left(p_{1}, p_{2}\right)}\right)=C_{r, s: m} \int_{0}^{\infty} \int_{y}^{\infty} y^{p_{1}} z^{p_{2}}[F(y)]^{r-1}[F(z)-F(y)]^{s-r-1}[1-F(z)]^{n-s} f(y) f(z) \mathrm{d} z \mathrm{~d} y .
$$

By using the same argument as in Theorem 1, we get the result given in (17).
2.3. Triple Moments. Let $Y_{1: m} \leq Y_{2: m} \leq \cdots \leq Y_{n: m}$ denote the order statistics drawn from the EPL distribution. The joint PDF of the $r$ th, $s$ th, and $t$ th order statistics is given by

$$
\begin{aligned}
& f_{Y_{(r: m)}, Y_{(s: m)}, Y_{(t: m)}}(y, z, u)=C_{r, s, t: m} F^{r-1}(y)[F(z)-F(y)]^{s-1-r}[F(u)-F(z)]^{t-1-s} \\
& \times[1-F(u)]^{m-t} f(y) f(z) f(u), \quad 0<y<z<u,
\end{aligned}
$$

where $1 \leq r<s<t \leq m$ and

$$
C_{r, s, t: m}=\frac{m !}{(r-1) !(s-r-1) !(t-s-1) !(m-t) !} .
$$

Theorem 3. For the EPL distribution given in (1), The triple moment of $r$ th, sth, and th order statistics $\mu_{r, s, t: m}^{\left(p_{1}, p_{2}, p_{3}\right)}$, $p_{1}, p_{2}, p_{3}=1,2, \ldots$, is as follows: 


$$
\begin{aligned}
\mu_{r, s, t: m}^{\left(p_{1}, p_{2}, p_{3}\right)}= & \delta^{3} C_{r, s, t: m} \sum_{\ell_{1}=0}^{s-1-r} \sum_{\ell_{2}=0}^{t-1-s} \sum_{\ell_{3}=0}^{m-t}\left(\begin{array}{c}
s-1-r \\
\ell_{1}
\end{array}\right)\left(\begin{array}{c}
t-1-s \\
\ell_{2}
\end{array}\right)\left(\begin{array}{c}
m-t \\
\ell_{3}
\end{array}\right)(-1)^{\ell_{1}+\ell_{2}+\ell_{3}} \\
& \times \sum_{j_{1}=0}^{\delta\left(t-s-\ell_{2}+\ell_{3}\right)-1} \sum_{j_{2}=0}^{j_{1}} \sum_{j_{3}=0}^{j_{2}+1} \sum_{j_{4}=0}^{\left(p_{3} / \gamma\right)+j_{3} \delta\left(s-r-\ell_{1}+\ell_{2}\right)-1} \sum_{j_{5}=0}^{j_{5}} \sum_{j_{6}=0}^{j_{j_{7}}=0} \sum_{j_{8}=0}^{j_{6}+1} \sum_{j_{9}=0}^{\left(p_{2} / \gamma\right)+j_{4}+j_{7} \delta\left(r+\ell_{1}\right)-1} \sum_{j_{10}=0}^{j_{9}} \sum_{j_{11}=0}^{j_{10}+1} \\
& \times\left(\begin{array}{c}
\delta\left(t-s-\ell_{2}+\ell_{3}\right)-1 \\
j_{1} \\
j_{2}
\end{array}\right)\left(\begin{array}{c}
j_{1} \\
j_{2}
\end{array}\right)\left(\begin{array}{c}
j_{2}+1 \\
j_{3}
\end{array}\right)\left(\begin{array}{c}
\left.\delta-r-\ell_{1}+\ell_{2}\right)-1 \\
j_{6}
\end{array}\right)\left(\begin{array}{c}
j_{6}+1 \\
j_{7}
\end{array}\right)\left(\begin{array}{c}
j_{10}+1 \\
j_{10}
\end{array}\right) \frac{(-1)^{j_{1}+j_{5}+j_{9}} v^{j_{2}-j_{3}+j_{6}-j_{7}+j_{10}-j_{11}-\left(\left(p_{1}+p_{2}+p_{3}\right) / \gamma\right)+3}}{j_{4} ! j_{8} !(1+v)^{j_{1}+j_{5}+j_{9}+3}\left(j_{1}+1\right)^{(l / \beta)+j_{3}-j_{4}+1}} \\
& \times\left(\begin{array}{c}
\delta\left(r+\ell_{1}\right)-1 \\
j_{11}
\end{array}\right. \\
& \times \frac{\left(\left(p_{3} / \gamma\right)+j_{3}\right) !\left(\left(p_{2} / \gamma\right)+j_{4}+j_{7}\right) ! \Gamma\left(\left(p_{1} / \gamma\right)+j_{8}+j_{11}+1\right)}{\left(j_{5}+j_{1}+2\right)} .
\end{aligned}
$$

Proof. From equation (19), we have

$$
\begin{aligned}
\mu_{r, s, t: m}^{\left(p_{1}, p_{2}, p_{3}\right)}=E\left(Y_{r: m}^{\left(p_{1}\right)} Y_{s: m}^{\left(p_{2}\right)} Y_{t: m}^{\left(p_{3}\right)}\right)= & C_{r, s, t: m} \int_{0}^{\infty} \int_{y}^{\infty} \int_{z}^{\infty} y^{p_{1}} z^{p_{2}} u^{p_{3}} F^{r-1}(y)[F(z)-F(y)]^{s-r-1} \\
& \times[F(u)-F(z)]^{t-s-1}[1-F(u)]^{m-t} f(y) f(z) f(u) \mathrm{d} u \mathrm{~d} z \mathrm{~d} y .
\end{aligned}
$$

By using the same argument as in Theorems 1 and 2, we obtain the result given in (21).
2.4. Quadruple Moments. Let $Y_{1: m} \leq Y_{2: m} \leq \cdots \leq Y_{n: m}$ denote the order statistics drawn from the EPL distribution. The joint PDF of the $r$ th, $s$ th, $t$ th, and $w$ th order statistics is given as

$$
\begin{aligned}
f_{Y_{(r: m)}, Y_{(s: m)}, Y_{(t: m)}, Y_{(w: m)}}(y, z, u, v)= & C_{r, s, t, w: m} F^{r-1}(y)[F(z)-F(y)]^{s-1-r}[F(u)-F(z)]^{t-1-s} \\
& \times[F(v)-F(u)]^{w-1-t}[1-F(v)]^{m-w} f(y) f(z) f(u) f(v),
\end{aligned}
$$

where $1 \leq r<s<t<u \leq m$ and

$$
C_{r, s, t, w: m}=\frac{m !}{(r-1) !(s-r-1) !(t-s-1) !(w-t-1)(m-w) !} .
$$

Theorem 4. For the EPL distribution given in (1), the quadruple moment of $r$ th, sth, th, and wth order statistics $\mu_{r, s, t: m}^{\left(p_{1}, p_{2}, p_{3}, p_{4}\right)}$ is as follows: 


$$
\begin{aligned}
& \mu_{r, s, t: m}^{\left(p_{1}, p_{2}, p_{3}, p_{4}\right)}=\delta^{4} C_{r, s, t, u: m} \sum_{\ell_{1}=0}^{s-1-r} \sum_{\ell_{2}=0}^{t-1-s} \sum_{\ell_{3}=0}^{u-1-t} \sum_{\ell_{4}=0}^{m-u}\left(\begin{array}{c}
s-1-r \\
\ell_{1}
\end{array}\right)\left(\begin{array}{c}
t-1-s \\
\ell_{2}
\end{array}\right)\left(\begin{array}{c}
u-1-t \\
\ell_{3}
\end{array}\right)\left(\begin{array}{c}
m-u \\
\ell_{4}
\end{array}\right) \\
& \times(-1)^{\ell_{1}+\ell_{2}+\ell_{3}+\ell_{4}} \sum_{j_{1}=0}^{\delta\left(w-t-\ell_{3}+\ell_{4}\right)-1} \sum_{j_{2}=0}^{j_{1}} \sum_{j_{3}=0}^{j_{2}+1} \sum_{j_{4}=0}^{\left(p_{4} / \gamma\right)+j_{3} \delta\left(t-s-\ell_{2}+\ell_{3}\right)-1} \sum_{j_{5}=0}^{j_{5}} \sum_{j_{6}=0}^{j_{6}+1} \sum_{j_{7}=0}^{j_{j_{8}}=0} \sum_{\left.p_{3} / \gamma\right)+j_{4}+j_{7}} \\
& \times \sum_{j_{9}=0}^{\delta\left(s-r-\ell_{1}+\ell_{2}\right)-1} \sum_{j_{10}=0}^{j_{9}} \sum_{j_{11}=0}^{j_{10}+1} \sum_{i_{12}=0}^{\left(p_{2} / \gamma\right)+j_{8}+j_{11}} \sum_{j_{13}=0}^{\delta\left(r+\ell_{1}\right)-1} \sum_{j_{14}=0}^{j_{13}} \sum_{j_{15}=0}^{i_{14+1}}\left(\begin{array}{c}
\delta\left(w-t-\ell_{3}+\ell_{4}\right)-1 \\
j_{1}
\end{array}\right) \\
& \times\left(\begin{array}{c}
j_{1} \\
j_{2}
\end{array}\right)\left(\begin{array}{c}
j_{2}+1 \\
j_{3}
\end{array}\right)\left(\begin{array}{c}
\delta\left(t-s-\ell_{2}+\ell_{3}\right)-1 \\
j_{5}
\end{array}\right)\left(\begin{array}{c}
j_{5} \\
j_{6}
\end{array}\right)\left(\begin{array}{c}
j_{6}+1 \\
j_{7}
\end{array}\right)\left(\begin{array}{c}
\delta\left(s-r-\ell_{1}+\ell_{2}\right)-1 \\
j_{9}
\end{array}\right) \\
& \times\left(\begin{array}{c}
j_{9} \\
j_{10}
\end{array}\right)\left(\begin{array}{c}
j_{10}+1 \\
j_{11}
\end{array}\right)\left(\begin{array}{c}
\delta\left(r+\ell_{1}\right)-1 \\
j_{13}
\end{array}\right)\left(\begin{array}{c}
j_{13} \\
j_{14}
\end{array}\right)\left(\begin{array}{c}
j_{14}+1 \\
j_{15}
\end{array}\right) \\
& \times \frac{(-1)^{j_{1}+j_{5}+j_{9}+j_{13}} v^{j_{2}-j_{3}+j_{6}-j_{7}+j_{10}-j_{11}+j_{14}-j_{15}-\left(\left(p_{1}+p_{2}+p_{3}+p_{4}\right) / \gamma\right)+4}}{j_{4} ! j_{8} ! j_{12} !(1+v)^{j_{1}+j_{5}+j_{9}+j_{13}+4}\left(j_{1}+1\right)^{\left(p_{4} / \gamma\right)+j_{3}-j_{4}+1}} \\
& \times \frac{\left(\left(p_{4} / \gamma\right)+j_{3}\right) !\left(\left(p_{3} / \gamma\right)+j_{4}+j_{7}\right) !\left(\left(p_{2} / \gamma\right)+j_{8}+j_{11}\right) !}{\left(j_{5}+j_{1}+2\right)^{\left(p_{4} / \gamma\right)+j_{4}+j_{7}-j_{8}+1}\left(j_{9}+j_{5}+j_{1}+3\right)^{\left(p_{2} / \gamma\right)+j_{8}+j_{11}-j_{12}+1}} \\
& \times \frac{\Gamma\left(\left(p_{1} / \gamma\right)+j_{12}+j_{15}+1\right)}{\left(j_{13}+j_{9}+j_{5}+j_{1}+4\right)^{\left(p_{1} / \gamma\right)+j_{12}+j_{15}+1}} .
\end{aligned}
$$

Proof. From equation (23), we have

$$
\begin{aligned}
\mu_{r, s, t: m}^{\left(p_{1}, p_{2}, p_{3}, p_{4}\right)}= & E\left(Y_{r: m}^{\left(p_{1}\right)} Y_{s: m}^{\left(p_{2}\right)} Y_{t: m}^{\left(p_{3}\right)} Y_{w: m}^{\left(p_{4}\right)}\right) \\
= & C_{r, s, t, w: m} \int_{0}^{\infty} \int_{y}^{\infty} \int_{z}^{\infty} \int_{u}^{\infty} \\
& \cdot y^{p_{1}} z^{p_{2}} u^{p_{3}} v^{p_{4}}[F(z)-F(y)]^{s-1-r} \\
& \times F^{r-1}(y)[F(u)-F(z)]^{t-1-s} \\
& \cdot[F(v)-F(u)]^{w-1-t}[1-F(v)]^{m-w} \\
& \cdot f(y) f(v) f(u) f(v) \mathrm{d} v \mathrm{~d} u \mathrm{~d} z \mathrm{~d} y .
\end{aligned}
$$

By using the same argument as in Theorem 3, we get the result given in (25).

\section{Estimation of Parameters}

Here, we study parameter estimation for the EPL distribution based on order statistics.

3.1. BLUEs of Parameters. The PDF of the scale-parameter EPL distribution is

$$
\begin{aligned}
f(y)= & \frac{\delta \gamma v^{2}}{\sigma(1+v)}\left(\frac{y}{\sigma}\right)^{\gamma-1}\left[1+\left(\frac{y}{\sigma}\right)^{\gamma}\right] \\
& \cdot\left[1-\frac{1+v+v(y / \sigma)^{\gamma}}{1+v} e^{-v(y / \sigma)^{\gamma}}\right]^{\delta-1} \\
& \cdot e^{-v(y / \sigma)^{\gamma}}, \quad y>0,(\delta, \gamma, v, \sigma)>0,
\end{aligned}
$$

and the PDF of the location-scale parameter EPL distribution is

$$
\begin{aligned}
f(y)= & \frac{\delta \gamma v^{2}}{\sigma(1+v)}\left(\frac{y-\mu}{\sigma}\right)^{\gamma-1}\left[1+\left(\frac{y-\mu}{\sigma}\right)^{\gamma}\right] e^{-v((y-\mu) / \sigma)^{\gamma}} \\
\times & {\left[1-\frac{1+v+v((y-\mu) / \sigma)^{\gamma}}{1+v} e^{-v((y-\mu) / \sigma)^{\gamma}}\right]^{\delta-1}, } \\
& y>0,(\delta, \gamma, v, \sigma, \mu)>0 .
\end{aligned}
$$

Let $Y_{1: m} \leq Y_{2: m} \leq \cdots \leq Y_{n-c: m}, c=0,1, \ldots, n-1$ denote type-II right-censored sample from the location-scale parameter EPL distribution in equation (1). Let us denote $X_{r: m}=\left(Y_{r: m}-\mu\right) / \sigma, E\left(X_{r: m}\right)=\mu_{r: m}^{(1)}, 1 \leq r \leq(m-c)$, and $\operatorname{Cov}\left(X_{r: m}, X_{s: m}\right)=\sigma_{r, s: m}=\mu_{r, s: m}^{(1,1)}-\mu_{r: m}^{(1)} \mu_{s: m}^{(1)}$, $1 \leq r<s \leq(m-c)$. Therefore, 


$$
\begin{aligned}
\mathbf{Y} & =\left(Y_{1: m}, Y_{2: m}, \ldots, Y_{(m-c): m}\right)^{T}, \\
\boldsymbol{\mu} & =\left(\mu_{1: m}, \mu_{2: m}, \ldots, \mu_{(m-c): m}\right)^{T}, \\
1 & =\underbrace{(1,1, \ldots, 1)^{T}}_{m-c}, \\
\sum & =\left(\left(\sigma_{r, s}\right)\right), \quad 1 \leq r, s \leq m-c,
\end{aligned}
$$

where $\quad \mu_{i: m}=E\left(Y_{i: m}\right), \quad \sigma_{i i}=\operatorname{Var}\left(Y_{i: m}\right) \quad$ and $\sigma_{i j}=\operatorname{Cov}\left(Y_{i: m}, Y_{j: m}\right)$, and $i=1,2, \ldots(m-c)$. Then, the BLUEs of $\mu$ and $\sigma$ can be computed as follows (see Arnold et al. [8]):

$$
\begin{aligned}
\mu^{*} & =\sum_{r=1}^{m-c} p_{r} Y_{r: m}, \\
\sigma^{*} & =\sum_{r=1}^{m-c} q_{r} Y_{r: m}
\end{aligned}
$$

where

$$
\begin{aligned}
& p_{r}=\left\{\frac{\mu^{T} \Sigma^{-1} \mu 1^{T} \sum^{-1}-\mu^{T} \Sigma^{-1} 1 \mu^{T} \Sigma^{-1}}{\left(\mu^{T} \Sigma^{-1} \mu\right)\left(1^{T} \Sigma^{-1} 1\right)-\left(\mu^{T} \Sigma^{-1} 1\right)^{2}}\right\} \\
& q_{r}=\left\{\frac{1^{T} \sum^{-1} 1 \mu^{T} \sum^{-1}-1^{T} \sum^{-1} \mu 1^{T} \Sigma^{-1}}{\left(\mu^{T} \sum^{-1} \mu\right)\left(1^{T} \Sigma^{-1} 1\right)-\left(\mu^{T} \Sigma^{-1} 1\right)^{2}}\right\} .
\end{aligned}
$$

The variances and covariance of these BLUEs can be computed as follows (see Arnold et al. [8]):

$$
\begin{array}{r}
\operatorname{Var}\left(\mu^{*}\right)=\sigma^{2}\left\{\frac{\mu^{T} \sum^{-1} \mu}{\left(\mu^{T} \Sigma^{-1} \mu\right)\left(1^{T} \Sigma^{-1} 1\right)-\left(\mu^{T} \Sigma^{-1} 1\right)^{2}}\right\}=\sigma^{2} W_{1}, \\
\operatorname{Var}\left(\sigma^{*}\right)=\sigma^{2}\left\{\frac{1^{T} \Sigma^{-1} 1}{\left(\mu^{T} \Sigma^{-1} \mu\right)\left(1^{T} \Sigma^{-1} 1\right)-\left(\mu^{T} \Sigma^{-1} 1\right)^{2}}\right\}=\sigma^{2} W_{2}, \\
\operatorname{Cov}\left(\mu^{*}, \sigma^{*}\right)=\sigma^{2}\left\{\frac{-\mu^{T} \Sigma^{-1} 1}{\left(\mu^{T} \Sigma^{-1} \mu\right)\left(1^{T} \Sigma^{-1} 1\right)-\left(\mu^{T} \Sigma^{-1} 1\right)^{2}}\right\}=\sigma^{2} W_{3} .
\end{array}
$$

The values of $p_{r}$ and $q_{r}$ are displayed in Tables 1 and 2 for different values of sample sizes $n=7,10$ and different censoring cases $c=0(1)([n / 2]-1)$ and for some selected values for $v=1,2$. The coefficient of the BLUEs $p_{r}$ and $q_{r}$ are given by (17) and (18), respectively, with conditions

$$
\begin{aligned}
& \sum_{i=1}^{n-c} p_{i}=1, \\
& \sum_{i=1}^{n-c} q_{i}=0,
\end{aligned}
$$

which are used to check the computations accuracy.

\section{Approximate Inference}

Here, we derive the $(1-\alpha) 100 \%$ confidence intervals for the parameters $\mu$ and $\sigma$ based on the following pivotal quantities:

$$
\begin{aligned}
& U_{1}=\frac{\mu^{*}-\mu}{\sigma \sqrt{W_{1}}}, \\
& U_{2}=\frac{\sigma^{*}-\sigma}{\sigma \sqrt{W_{2}}} \\
& U_{3}=\frac{\mu^{*}-\mu}{\sigma^{*} \sqrt{W_{1}}},
\end{aligned}
$$

where $\mu^{*}$ and $\sigma^{*}$ are the BLUEs of $\mu$ and $\sigma$ with variances $\sigma^{2} W_{1}$ and $\sigma^{2} W_{2}$, respectively. $U_{1}$ is used to draw inference for $\mu$ when $\sigma$ is known, while $U_{3}$ can be used to draw inference for $\mu$ when $\sigma$ is unknown. Similarly, $U_{2}$ can be used to draw inference for $\sigma$ when $\mu$ is unknown.

The moments presented in Section 2 are used to derive the confidence intervals of the location and scale parameters based on the pivotal quantities in equation (35).

Hence, $U_{1}$ and $U_{2}$ can be rewritten as

$$
\begin{aligned}
& U_{1}=\frac{1}{\sqrt{W_{1}}}\left(\sum_{i=1}^{m-c} p_{i} X_{i: m}\right)=\frac{U_{1}^{*}}{\sqrt{W_{1}}} \\
& U_{2}=\frac{1}{\sqrt{W_{2}}}\left(\sum_{i=1}^{m-c} q_{i} X_{i: m}-1\right)=\frac{U_{2}^{*}-1}{\sqrt{W_{2}}},
\end{aligned}
$$

where $X_{i: m}=\left(Y_{i: m}-\mu\right) / \sigma, i=1,2, \ldots, m-c$, is the standardized form of the available type-II right-censored sample $Y_{i: m}, i=1,2, \ldots, m-c$.

we consider finding the approximate distribution by using Edgeworth approximation for a statistic $\mathrm{S}$ (with mean 0 and variance 1 ) as

$$
\begin{aligned}
H(s) \approx & \Phi(s)-\phi(s)\left[\frac{\sqrt{\alpha_{1}}}{6}\left(s^{2}-1\right)+\frac{\alpha_{2}-3}{24}\left(s^{3}-3 s\right)\right. \\
& \left.+\frac{\alpha_{1}}{72}\left(s^{5}-10 s^{2}+15 s\right)\right],
\end{aligned}
$$

where $\sqrt{\alpha_{1}}$ and $\alpha_{2}$ are the coefficients of skewness and kurtosis of $S$, respectively, and $\Phi(s)$ and $\phi(s)$ are the CDF and PDF of the standard normal distribution, respectively.

\section{Numerical Aspects}

The relations obtained in the preceding sections allow us to evaluate coefficients of the BLUEs. The coefficients of the BLUEs are presented in Tables 1 and 2 while the variances and covariances of the BLUEs are presented in Table 3. From the results presented in Table 3, we observe that the variance of the BLUEs increases as the censoring level increases while the variance of the BLUEs decreases when the sample size increases and increases as $v$ increases. In addition, we observe that the covariances of the BLUEs decrease as the censoring level increases while the covariances of the BLUEs increase when the sample size increases and decrease as $v$ increases.

Tables 4-6 display the simulated percentage points at $v=$ 1 (1) 3 and sample size $n=7,10$. Table 7 presents the values of the mean, variance, and coefficient of skewness and 
TABLE 1: Coefficients of the BLUEs of the location parameter.

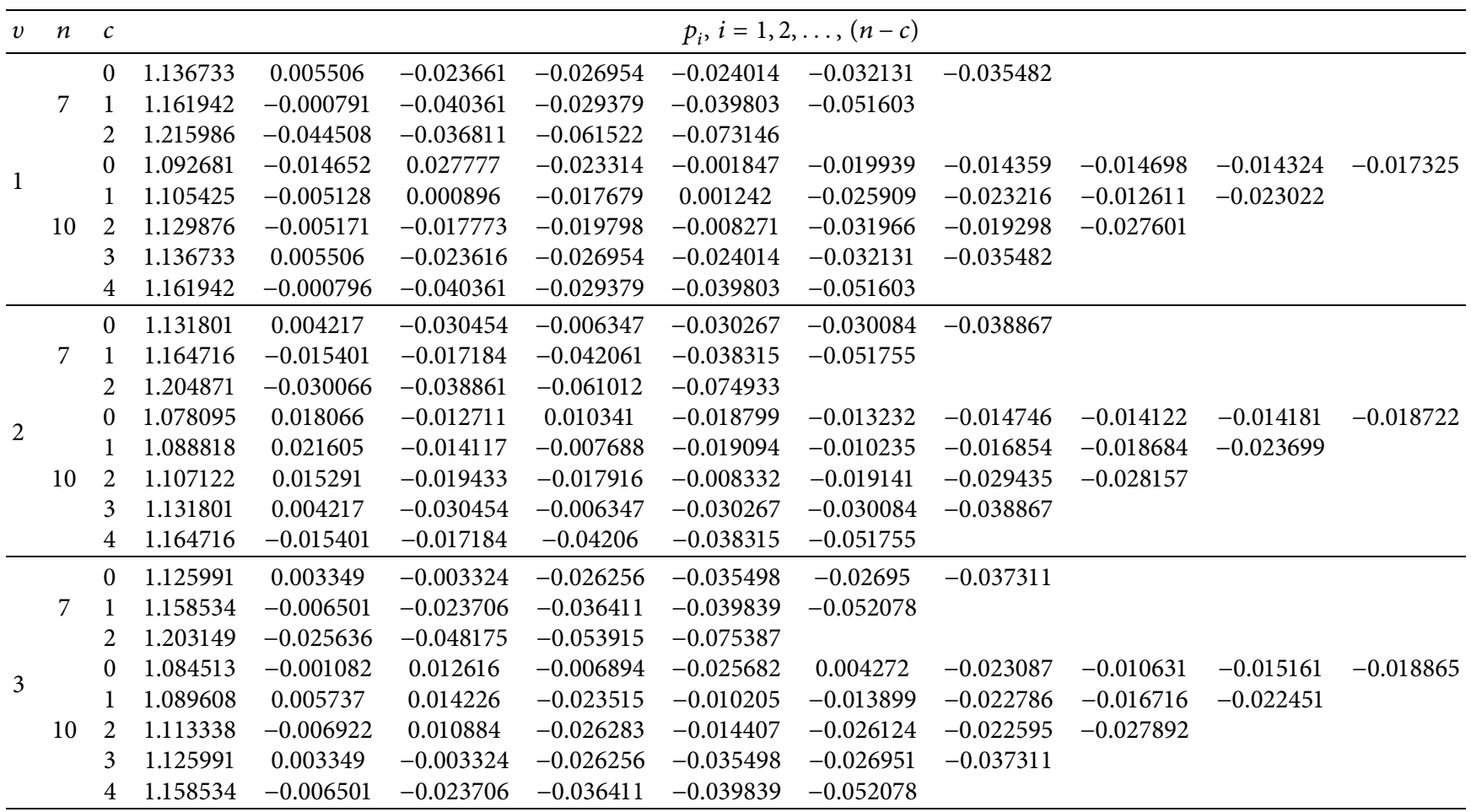

TABLE 2: Coefficients of the BLUEs of the scale parameter.

\begin{tabular}{|c|c|c|c|c|c|c|c|c|c|c|c|c|}
\hline$v$ & $n$ & $c$ & & & & & $q_{i}, i=1,2$, &.,$(n-c)$ & & & & \\
\hline \multirow{8}{*}{1} & \multirow{3}{*}{7} & 0 & -0.607191 & 0.049265 & 0.107357 & 0.098563 & 0.106076 & 0.115131 & 0.130798 & & & \multirow{8}{*}{0.087283} \\
\hline & & 1 & -0.619096 & 0.065174 & 0.148212 & 0.116684 & 0.133273 & 0.155753 & & & & \\
\hline & & 2 & -0.650729 & 0.142376 & 0.160171 & 0.154025 & 0.194157 & & & & & \\
\hline & \multirow{5}{*}{10} & 0 & -0.615392 & 0.081514 & 0.028793 & 0.038189 & 0.074711 & 0.069523 & 0.077051 & 0.087084 & 0.071246 & \\
\hline & & 1 & -0.623159 & 0.089041 & 0.027265 & 0.079845 & 0.064478 & 0.087226 & 0.094728 & 0.082462 & 0.098115 & \\
\hline & & 2 & -0.610879 & 0.067192 & 0.042666 & 0.108053 & 0.081235 & 0.102664 & 0.097707 & 0.111363 & & \\
\hline & & 3 & -0.607191 & 0.049265 & 0.107357 & 0.098563 & 0.106076 & 0.115131 & 0.130798 & & & \\
\hline & & 4 & -0.619096 & 0.065174 & 0.148212 & 0.116684 & 0.133273 & 0.155753 & & & & \\
\hline \multirow{8}{*}{2} & \multirow{3}{*}{7} & 0 & -0.603985 & 0.056041 & 0.086529 & 0.098091 & 0.120122 & 0.117091 & 0.126112 & & \multirow{8}{*}{$\begin{array}{l}0.081904 \\
0.096037\end{array}$} & \multirow{8}{*}{0.085201} \\
\hline & & 1 & -0.624402 & 0.081701 & 0.122939 & 0.128854 & 0.140442 & 0.150465 & & & & \\
\hline & & 2 & -0.647063 & 0.145811 & 0.136075 & 0.176638 & 0.188541 & & & & & \\
\hline & \multirow{5}{*}{10} & 0 & -0.575495 & 0.011332 & 0.070903 & 0.025755 & 0.098121 & 0.040347 & 0.079868 & 0.082063 & & \\
\hline & & 1 & -0.580427 & 0.010583 & 0.078087 & 0.062771 & 0.081424 & 0.065365 & 0.098493 & 0.087668 & & \\
\hline & & 2 & -0.598187 & 0.043684 & 0.069971 & 0.095037 & 0.077136 & 0.097313 & 0.106356 & \multirow{3}{*}{0.108691} & & \\
\hline & & 3 & -0.603985 & 0.056041 & 0.086529 & 0.098091 & 0.120122 & 0.117091 & 0.126112 & & & \\
\hline & & 4 & -0.624402 & 0.081701 & 0.122939 & 0.128854 & 0.140442 & 0.150465 & & & & \\
\hline \multirow{8}{*}{3} & & 0 & -0.610973 & 0.071403 & 0.064731 & 0.127295 & 0.117376 & 0.097999 & 0.132169 & & & \multirow{8}{*}{0.091304} \\
\hline & \multirow[t]{2}{*}{7} & 1 & -0.635591 & 0.101063 & 0.121287 & 0.132771 & 0.123643 & 0.156826 & & & & \\
\hline & & 2 & -0.635278 & 0.123788 & 0.159474 & 0.157116 & 0.194901 & & & & & \\
\hline & \multirow{5}{*}{10} & 0 & -0.608131 & 0.067851 & 0.040462 & 0.026115 & 0.087299 & 0.069354 & 0.083118 & 0.079318 & 0.063311 & \\
\hline & & 1 & -0.601453 & 0.054542 & 0.050085 & 0.045373 & 0.094679 & 0.099195 & 0.082039 & 0.075534 & 0.100006 & \\
\hline & & 2 & -0.605026 & 0.060417 & 0.060951 & 0.063863 & 0.126491 & 0.094747 & 0.085135 & 0.113422 & & \\
\hline & & 3 & -0.610973 & 0.071403 & 0.064731 & 0.127295 & 0.117376 & 0.097999 & 0.132169 & & & \\
\hline & & 4 & -0.635591 & 0.101063 & 0.121287 & 0.132771 & 0.123643 & 0.156826 & & & & \\
\hline
\end{tabular}

kurtosis $U_{1}^{*}$ and $U_{2}^{*}$. The simulated average widths of confidence intervals are presented in Table 8 . We observe that the Edgeworth approximations of the distributions of
$U_{1}$ and $U_{2}$ both work quite satisfactorily; this is also clear from the average width of the confidence intervals based on $U_{1}$ and $U_{2}$ which are presented in Table 8 . 
TABLE 3: Variance and covariance of the BLUEs when $\mu=0$ and $\sigma=1$.

\begin{tabular}{|c|c|c|c|c|c|}
\hline$v$ & $n$ & $c$ & $\operatorname{Var}(\mu)$ & $\operatorname{Var}(\sigma)$ & $\operatorname{Cov}(\mu, \sigma)$ \\
\hline \multirow{8}{*}{1} & \multirow{3}{*}{7} & 0 & 0.068821 & 0.135051 & -0.041267 \\
\hline & & 1 & 0.093333 & 0.160655 & -0.055683 \\
\hline & & 2 & 0.141613 & 0.201843 & -0.083785 \\
\hline & \multirow{5}{*}{10} & 0 & 0.033913 & 0.088141 & -0.020113 \\
\hline & & 1 & 0.042059 & 0.100032 & -0.024623 \\
\hline & & 2 & 0.053405 & 0.116162 & -0.031974 \\
\hline & & 3 & 0.068821 & 0.135051 & -0.041267 \\
\hline & & 4 & 0.093333 & 0.160655 & -0.055683 \\
\hline & \multirow{3}{*}{7} & 0 & 0.070562 & 0.135301 & -0.041106 \\
\hline & & 1 & 0.098388 & 0.164369 & -0.057362 \\
\hline & & 2 & 0.146701 & 0.208599 & -0.085694 \\
\hline & \multirow{5}{*}{10} & 0 & 0.034945 & 0.089757 & -0.020554 \\
\hline 2 & & 1 & 0.042393 & 0.101301 & -0.025082 \\
\hline & & 2 & 0.054041 & 0.114633 & -0.030894 \\
\hline & & 3 & 0.070562 & 0.135301 & -0.041106 \\
\hline & & 4 & 0.098388 & 0.164369 & -0.057362 \\
\hline \multirow{8}{*}{3} & \multirow{3}{*}{7} & 0 & 0.072062 & 0.137961 & -0.041643 \\
\hline & & 1 & 0.100875 & 0.167103 & -0.058229 \\
\hline & & 2 & 0.142707 & 0.211536 & -0.084917 \\
\hline & \multirow{5}{*}{10} & 0 & 0.033831 & 0.091732 & -0.020271 \\
\hline & & 1 & 0.042178 & 0.103774 & -0.025141 \\
\hline & & 2 & 0.053543 & 0.119151 & -0.031737 \\
\hline & & 3 & 0.072062 & 0.137961 & -0.041643 \\
\hline & & 4 & 0.100875 & 0.167103 & -0.058229 \\
\hline
\end{tabular}

\section{Real Data Analysis}

In this section, we analyze two real data sets to show the importance of the proposed estimators. One data set from environmental monitoring and another data is from maximum flood level for the Susquehanna River at Harrisburg.
Example 1. Analysis of clean upgradient ground-water monitoring wells subjected to $\mathrm{mg} / \mathrm{L}$.In the first data set, we consider vinyl chloride data obtained from clean upgradient monitoring wells which was studied by many authors such as Bhaumik and Gibbons [26]; Krishnamoorthy et al. [27]; Bhaumik et al. [28]; and Kumar and Goyal $[22,23]$. The data are as follows:

$$
\begin{array}{ccccccccccccccccc}
5.1 & 1.2 & 1.3 & 0.6 & 0.5 & 2.4 & 0.5 & 1.1 & 8.0 & 0.8 & 0.4 & 0.6 & 0.9 & 0.4 & 2.0 & 0.5 & 5.3 \\
3.2 & 2.7 & 2.9 & 2.5 & 2.3 & 1.0 & 0.2 & 0.1 & 0.1 & 1.8 & 0.9 & 2.0 & 4.0 & 6.8 & 1.2 & 0.4 & 0.2 \text {. }
\end{array}
$$

Now, a random sample of size 10 is selected from the given data set 1.0, 1.2, 3.2, 2.4, 0.8, 2.0, 0.4, 0.2, 2.9, 1.2. By the EPL distribution for the given sample, we have obtained the maximum likelihood estimate of $\delta_{\mathrm{ML}}=0.59023, \gamma_{\mathrm{ML}}=$ 1.808770 , and $v_{\mathrm{ML}}=0.434371$. By computation, the Kolmogorov-Smirnov $(\mathrm{K}-\mathrm{S})$ distance and the corresponding $p$ values are 0.163772 and 0.9513 , respectively, which implies that the EPL distribution provides a reasonable model for this data. Moveover, the empirical cumulative distribution function (ECDF) plot and the Quantile-Quantile (Q-Q) plots are also presented under the vinyl chloride data as shown in Figure 1, which also imply that the EPL distribution can be used as a proper model to fit this data.
Then, by using the BLUE coefficients in Tables 1 and 2, we have

$$
\begin{aligned}
\mu^{*} & =\sum_{j=1}^{n} p_{j} X_{j: n}=0.024467 \\
\sigma^{*} & =\sum_{j=1}^{n} q_{j} X_{j: n}=0.992854
\end{aligned}
$$

Example 2. Analysis of the maximum flood level for the Susquehanna River at Harrisburg, Pennsylvania.The second data set presents the maximum flood level for the Susquehanna River at Harrisburg, Pennsylvania, and it was studied by Dumonceaux and Antle [29]. The data are

$$
\begin{array}{cccccccccc}
0.654 & 0.613 & 0.315 & 0.449 & 0.297 & 0.402 & 0.379 & 0.423 & 0.379 & 0.3235 \\
0.269 & 0.740 & 0.418 & 0.412 & 0.494 & 0.416 & 0.338 & 0.392 & 0.484 & 0.265 \text {. }
\end{array}
$$


TABLE 4: Simulated values of the distribution of $U_{1}$ when $\mu=0$ and $\sigma=1$.

\begin{tabular}{|c|c|c|c|c|c|c|c|c|c|c|}
\hline$v$ & $n$ & $c$ & $1 \%$ & $2.50 \%$ & $5 \%$ & $10 \%$ & $90 \%$ & $95 \%$ & $97.50 \%$ & $99 \%$ \\
\hline \multirow{8}{*}{1} & \multirow{3}{*}{7} & 0 & -3.686292 & -3.686298 & -3.68631 & -1.23409 & 1.448849 & 1.811324 & 2.190922 & 3.049235 \\
\hline & & 1 & -1.754612 & -1.459681 & -1.25981 & -1.03561 & 1.307655 & 1.924963 & 2.499715 & 3.159464 \\
\hline & & 2 & -1.794954 & -1.508401 & -1.28934 & -1.05182 & 1.298412 & 1.895139 & 2.505579 & 3.260451 \\
\hline & \multirow{5}{*}{10} & 0 & -1.483964 & -1.326313 & -1.17203 & -0.99761 & 1.349227 & 1.938966 & 2.549851 & 3.414044 \\
\hline & & 1 & -1.525359 & -1.336776 & -1.18298 & -0.9978 & 1.343092 & 1.914571 & 2.540299 & 3.359295 \\
\hline & & 2 & -1.586863 & -1.376637 & -1.20636 & -1.01423 & 1.338138 & 1.922457 & 2.520371 & 3.232563 \\
\hline & & 3 & -1.630238 & -1.425475 & -1.23309 & -1.01737 & 1.311007 & 1.917257 & 2.517179 & 3.225972 \\
\hline & & 4 & -1.754612 & -1.459681 & -1.25981 & -1.03561 & 1.307655 & 1.924963 & 2.499715 & 3.159464 \\
\hline \multirow{8}{*}{2} & \multirow{3}{*}{7} & 0 & -1.615403 & -1.391141 & -1.21902 & -1.01619 & 1.319972 & 1.922683 & 2.491423 & 3.252833 \\
\hline & & 1 & -1.663496 & -1.448206 & -1.25977 & -1.03223 & 1.332836 & 1.918422 & 2.494936 & 3.207337 \\
\hline & & 2 & -1.802659 & -1.515264 & -1.29324 & -1.05587 & 1.319081 & 1.907491 & 2.448389 & 3.115657 \\
\hline & \multirow{5}{*}{10} & 0 & -1.479384 & -1.299185 & -1.15835 & -0.98451 & 1.343612 & 1.984078 & 2.548237 & 3.182261 \\
\hline & & 1 & -1.505824 & -1.348861 & -1.17004 & -0.99502 & 1.335991 & 1.955258 & 2.533781 & 3.205181 \\
\hline & & 2 & -1.548062 & -1.357995 & -1.18398 & -1.00052 & 1.346091 & 1.950572 & 2.496046 & 3.253751 \\
\hline & & 3 & -1.615403 & -1.391141 & -1.21902 & -1.01619 & 1.319972 & 1.922683 & 2.491423 & 3.252833 \\
\hline & & 4 & -1.663496 & -1.448206 & -1.25977 & -1.03223 & 1.332836 & 1.918422 & 2.494936 & 3.207337 \\
\hline \multirow{8}{*}{3} & \multirow{3}{*}{7} & 0 & -1.638623 & -1.422331 & -1.23184 & -1.01266 & 1.280785 & 1.903994 & 2.515901 & 3.268596 \\
\hline & & 1 & -1.709657 & -1.464361 & -1.24497 & -1.01847 & 1.276936 & 1.923978 & 2.499509 & 3.192122 \\
\hline & & 2 & -1.805743 & -1.519798 & -1.30221 & -1.05218 & 1.299622 & 1.909421 & 2.457119 & 3.214435 \\
\hline & \multirow{5}{*}{10} & 0 & -1.519226 & -1.329914 & -1.17265 & -1.00041 & 1.324002 & 1.937141 & 2.541251 & 3.326594 \\
\hline & & 1 & -1.550921 & -1.350977 & -1.18953 & -1.00624 & 1.293752 & 1.912262 & 2.554841 & 3.373878 \\
\hline & & 2 & -1.604826 & -1.395428 & -1.21302 & -1.0188 & 1.294612 & 1.921591 & 2.512003 & 3.376578 \\
\hline & & 3 & -1.638623 & -1.422331 & -1.23184 & -1.01266 & 1.280785 & 1.903994 & 2.515901 & 3.268596 \\
\hline & & 4 & -1.709657 & -1.464361 & -1.24497 & -1.01847 & 1.276936 & 1.923978 & 2.499509 & 3.192122 \\
\hline
\end{tabular}

TABLE 5: Simulated values of the distribution of $U_{2}$ when $\mu=0$ and $\sigma=1$.

\begin{tabular}{|c|c|c|c|c|c|c|c|c|c|c|}
\hline$v$ & $n$ & $c$ & $1 \%$ & $2.50 \%$ & $5 \%$ & $10 \%$ & $90 \%$ & $95 \%$ & $97.50 \%$ & $99 \%$ \\
\hline \multirow{8}{*}{1} & \multirow{3}{*}{7} & 0 & -2.678889 & -2.471173 & -2.301446 & -2.053213 & 0.454372 & 0.957141 & 1.421979 & 1.948598 \\
\hline & & 1 & -2.554143 & -2.375446 & -2.212094 & -1.974706 & 0.526451 & 1.017625 & 1.504291 & 2.077757 \\
\hline & & 2 & -2.395787 & -2.253001 & -2.077632 & -1.873422 & 0.630571 & 1.116881 & 1.612411 & 2.259385 \\
\hline & \multirow{5}{*}{10} & 0 & -3.019991 & -2.759098 & -2.548864 & -2.288165 & 0.237174 & 0.720949 & 1.085917 & 1.604703 \\
\hline & & 1 & -2.896556 & -2.664619 & -2.453476 & -2.199037 & 0.315051 & 0.800666 & 1.187191 & 1.704299 \\
\hline & & 2 & -2.807636 & -2.583198 & -2.374634 & -2.120514 & 0.392716 & 0.853461 & 1.299217 & 1.812966 \\
\hline & & 3 & -2.678889 & -2.471173 & -2.301446 & -2.053213 & 0.454372 & 0.957141 & 1.421979 & 1.948598 \\
\hline & & 4 & -2.554143 & -2.375446 & -2.212094 & -1.974706 & 0.526451 & 1.017625 & 1.504291 & 2.077757 \\
\hline \multirow{8}{*}{2} & \multirow{3}{*}{7} & 0 & -2.710056 & -2.505164 & -2.309474 & -2.070679 & 0.448602 & 0.949731 & 1.393545 & 1.913112 \\
\hline & & 1 & -2.582256 & -2.390288 & -2.218548 & -1.967492 & 0.523874 & 1.032095 & 1.508915 & 2.087237 \\
\hline & & 2 & -2.393321 & -2.230162 & -2.073431 & -1.862678 & 0.631468 & 1.147924 & 1.606034 & 2.213561 \\
\hline & \multirow{5}{*}{10} & 0 & -3.024276 & -2.730335 & -2.541782 & -2.270712 & 0.275171 & 0.734777 & 1.150898 & 1.665428 \\
\hline & & 1 & -2.898164 & -2.655736 & -2.463116 & -2.217135 & 0.317773 & 0.801581 & 1.253501 & 1.728512 \\
\hline & & 2 & -2.823059 & -2.605918 & -2.400323 & -2.143586 & 0.369156 & 0.863872 & 1.325027 & 1.825864 \\
\hline & & 3 & -2.710056 & -2.505164 & -2.309474 & -2.070679 & 0.448602 & 0.949731 & 1.393545 & 1.913112 \\
\hline & & 4 & -2.582256 & -2.390288 & -2.218548 & -1.967492 & 0.523874 & 1.032095 & 1.508915 & 2.087237 \\
\hline \multirow{8}{*}{3} & \multirow{3}{*}{7} & 0 & -2.649813 & -2.466518 & -2.258944 & -2.041399 & 0.498609 & 0.972135 & 1.437702 & 1.937601 \\
\hline & & 1 & -2.519256 & -2.344106 & -2.173079 & -1.946906 & 0.568205 & 1.095691 & 1.556311 & 2.072295 \\
\hline & & 2 & -2.371208 & -2.198918 & -2.060219 & -1.834712 & 0.659611 & 1.176077 & 1.635401 & 2.248324 \\
\hline & \multirow{5}{*}{10} & 0 & -2.926982 & -2.724922 & -2.529415 & -2.255072 & 0.266124 & 0.745879 & 1.168265 & 1.687633 \\
\hline & & 1 & -2.829318 & -2.645105 & -2.454084 & -2.199288 & 0.346392 & 0.800395 & 1.295153 & 1.766573 \\
\hline & & 2 & -2.748096 & -2.559353 & -2.350791 & -2.123788 & 0.411725 & 0.895525 & 1.360834 & 1.909579 \\
\hline & & 3 & -2.641983 & -2.466518 & -2.258944 & -2.041399 & 0.498609 & 0.972135 & 1.437702 & 1.937601 \\
\hline & & 4 & -2.519256 & -2.344106 & -2.173079 & -1.946906 & 0.568205 & 1.095691 & 1.556311 & 2.072295 \\
\hline
\end{tabular}

Now, a random sample of size 10 is selected from the given data set $0.418,0.265,0.613,0.269,0.654,0.338,0.315$, $0.379,0.297,0.412$. By the EPL distribution for the given sample, we have obtained the maximum likelihood estimate of $\quad \delta_{\mathrm{ML}}=3479.9773640, \quad \gamma_{\mathrm{ML}}=0.484329, \quad$ and
$v_{\mathrm{ML}}=14.626781$. By computation, the Kolmogorov-Smirnov $(\mathrm{K}-\mathrm{S})$ distance and the corresponding $p$ values are 0.14282 and 0.9694, respectively, which implies that the EPL distribution provides a reasonable model for this data. Moreover, as a further illustration, the empirical cumulative 
TABLE 6: Simulated values of the distribution of $U_{3}$ when $\mu=0$ and $\sigma=1$.

\begin{tabular}{|c|c|c|c|c|c|c|c|c|c|c|}
\hline$v$ & $n$ & $c$ & $1 \%$ & $2.50 \%$ & $5 \%$ & $10 \%$ & $90 \%$ & $95 \%$ & $97.50 \%$ & $99 \%$ \\
\hline \multirow{8}{*}{1} & \multirow{3}{*}{7} & 0 & -1.291825 & -1.262013 & -1.219325 & -1.149467 & 2.222395 & 3.523847 & 4.940092 & 7.134944 \\
\hline & & 1 & -1.293906 & -1.257118 & -1.216151 & -1.140551 & 2.350343 & 3.765088 & 5.389301 & 7.852344 \\
\hline & & 2 & -1.252756 & -1.219676 & -1.181444 & -1.106255 & 2.545932 & 4.159147 & 6.076095 & 8.899025 \\
\hline & \multirow{5}{*}{10} & 0 & -1.323036 & -1.286828 & -1.242329 & -1.165381 & 2.110051 & 3.223612 & 4.498312 & 6.167443 \\
\hline & & 1 & -1.308061 & -1.270312 & -1.231131 & -1.161611 & 2.148975 & 3.262731 & 4.524937 & 6.631667 \\
\hline & & 2 & -1.294854 & -1.264969 & -1.227509 & -1.157487 & 2.252917 & 3.411704 & 4.704752 & 6.501364 \\
\hline & & 3 & -1.291825 & -1.262013 & -1.219325 & -1.149467 & 2.222395 & 3.523847 & 4.940092 & 7.134944 \\
\hline & & 4 & -1.293906 & -1.257118 & -1.216151 & -1.140551 & 2.350343 & 3.765088 & 5.389301 & 7.852344 \\
\hline \multirow{8}{*}{2} & \multirow{3}{*}{7} & 0 & -1.299635 & -1.254525 & -1.210859 & -1.140081 & 2.320086 & 3.584004 & 5.171739 & 7.060132 \\
\hline & & 1 & -1.269125 & -1.231214 & -1.188392 & -1.119388 & 2.477281 & 3.871941 & 5.405274 & 7.898144 \\
\hline & & 2 & -1.234267 & -1.201231 & -1.165376 & -1.094119 & 2.620875 & 4.125688 & 5.952571 & 8.910766 \\
\hline & \multirow{5}{*}{10} & 0 & -1.316707 & -1.279803 & -1.234296 & -1.155644 & 2.127752 & 3.257233 & 4.511755 & 5.975934 \\
\hline & & 1 & -1.318328 & -1.274231 & -1.231598 & -1.152525 & 2.158989 & 3.380708 & 4.554498 & 6.349453 \\
\hline & & 2 & -1.306309 & -1.266361 & -1.220428 & -1.142738 & 2.227079 & 3.486914 & 4.694881 & 6.876191 \\
\hline & & 3 & -1.299635 & -1.254525 & -1.210859 & -1.140081 & 2.320086 & 3.584004 & 5.171739 & 7.060132 \\
\hline & & 4 & -1.269125 & -1.231214 & -1.188392 & -1.119388 & 2.477281 & 3.871941 & 5.405274 & 7.898144 \\
\hline \multirow{8}{*}{3} & \multirow{3}{*}{7} & 0 & -1.289032 & -1.249364 & -1.210882 & -1.131658 & 2.240714 & 3.457987 & 4.917726 & 7.291664 \\
\hline & & 1 & -1.258874 & -1.224641 & -1.180973 & -1.103054 & 2.319861 & 3.713039 & 5.304917 & 8.216081 \\
\hline & & 2 & -1.243127 & -1.210756 & -1.171678 & -1.099442 & 2.521328 & 4.071925 & 6.071664 & 9.205739 \\
\hline & \multirow{5}{*}{10} & 0 & -1.340722 & -1.297027 & -1.250248 & -1.174011 & 2.080714 & 3.246739 & 4.459408 & 6.277229 \\
\hline & & 1 & -1.326197 & -1.285656 & -1.237163 & -1.155345 & 2.090644 & 3.291885 & 4.750722 & 6.487507 \\
\hline & & 2 & -1.313368 & -1.278006 & -1.229561 & -1.152197 & 2.173185 & 3.366241 & 4.764053 & 6.650645 \\
\hline & & 3 & -1.289032 & -1.249364 & -1.210882 & -1.131658 & 2.240714 & 3.457987 & 4.917726 & 7.291664 \\
\hline & & 4 & -1.258874 & -1.224641 & -1.180973 & -1.103054 & 2.319861 & 3.713039 & 5.304917 & 8.216081 \\
\hline
\end{tabular}

TABLe 7: Mean, variance, and coefficient of skewness and kurtosis of $U_{1}^{*}$ and $U_{2}^{*}$ when $\mu=0$ and $\sigma=1$.

\begin{tabular}{|c|c|c|c|c|c|c|c|c|c|c|}
\hline \multirow{2}{*}{$v$} & \multirow{2}{*}{$n$} & \multirow{2}{*}{$c$} & \multicolumn{4}{|c|}{$U_{1}$} & \multicolumn{4}{|c|}{$U_{2}$} \\
\hline & & & Mean & $W_{1}$ & $\sqrt{\alpha_{1}}$ & $\alpha_{2}$ & Mean & $W_{1}$ & $\sqrt{\alpha_{1}}$ & $\alpha_{2}$ \\
\hline \multirow{8}{*}{1} & \multirow{3}{*}{7} & 0 & 0 & 0.039518 & 1.293415 & 2.911714 & -0.869812 & 0.077551 & 0.742414 & 0.979527 \\
\hline & & 1 & 0 & 0.053594 & 1.192561 & 2.520105 & -0.797493 & 0.092252 & 0.779777 & 1.008172 \\
\hline & & 2 & 0 & 0.081318 & 1.120099 & 2.226028 & -0.711487 & 0.115903 & 0.855671 & 1.063987 \\
\hline & \multirow{5}{*}{10} & 0 & 0 & 0.019474 & 1.423328 & 3.072102 & -1.076674 & 0.050613 & 0.577731 & 0.656743 \\
\hline & & 1 & 0 & 0.024152 & 1.390529 & 2.929123 & -1.010657 & 0.057441 & 0.646992 & 0.849696 \\
\hline & & 2 & 0 & 0.030666 & 1.313859 & 2.779625 & -0.937868 & 0.066703 & 0.704326 & 0.996806 \\
\hline & & 3 & 0 & 0.039518 & 1.293415 & 2.911714 & -0.869812 & 0.077551 & 0.742414 & 0.979527 \\
\hline & & 4 & 0 & 0.053594 & 1.192561 & 2.520105 & -0.797493 & 0.092252 & 0.779777 & 1.008172 \\
\hline \multirow{8}{*}{2} & \multirow{3}{*}{7} & 0 & 0 & 0.040446 & 1.346119 & 2.959134 & -0.872235 & 0.077554 & 0.657331 & 0.621787 \\
\hline & & 1 & 0 & 0.056396 & 1.290511 & 2.950683 & -0.791361 & 0.094215 & 0.711686 & 0.724204 \\
\hline & & 2 & 0 & 0.084088 & 1.154996 & 2.724035 & -0.702471 & 0.119568 & 0.845766 & 1.076625 \\
\hline & \multirow{5}{*}{10} & 0 & 0 & 0.020031 & 1.520931 & 3.976296 & -1.070904 & 0.051448 & 0.563839 & 0.452005 \\
\hline & & 1 & 0 & 0.024299 & 1.464689 & 3.627171 & -1.008042 & 0.058065 & 0.589949 & 0.502475 \\
\hline & & 2 & 0 & 0.030976 & 1.446371 & 3.457844 & -0.947609 & 0.065707 & 0.625893 & 0.561158 \\
\hline & & 3 & 0 & 0.040446 & 1.346119 & 2.959134 & -0.872235 & 0.077554 & 0.657331 & 0.621787 \\
\hline & & 4 & 0 & 0.056396 & 1.290511 & 2.950683 & -0.791361 & 0.094215 & 0.711686 & 0.724204 \\
\hline \multirow{8}{*}{3} & \multirow{3}{*}{7} & 0 & 0 & 0.041336 & 1.406169 & 3.581796 & -0.862483 & 0.079136 & 0.678438 & 0.457074 \\
\hline & & 1 & 0 & 0.057864 & 1.338369 & 3.284062 & -0.783676 & 0.095853 & 0.757721 & 0.647221 \\
\hline & & 2 & 0 & 0.081859 & 1.167649 & 2.806261 & -0.696523 & 0.121341 & 0.870486 & 0.981312 \\
\hline & \multirow{5}{*}{10} & 0 & 0 & 0.019406 & 1.420998 & 3.140231 & -1.057713 & 0.052619 & 0.563401 & 0.372104 \\
\hline & & 1 & 0 & 0.024194 & 1.468318 & 3.741814 & -0.994451 & 0.059526 & 0.590185 & 0.356138 \\
\hline & & 2 & 0 & 0.030713 & 1.375474 & 3.216648 & -0.928065 & 0.068347 & 0.631802 & 0.412006 \\
\hline & & 3 & 0 & 0.041336 & 1.406169 & 3.581796 & -0.862483 & 0.079136 & 0.678438 & 0.457074 \\
\hline & & 4 & 0 & 0.057864 & 1.338369 & 3.284062 & -0.783676 & 0.095853 & 0.757721 & 0.647221 \\
\hline
\end{tabular}


TABLE 8: Average width of the simulated CIs.

\begin{tabular}{|c|c|c|c|c|c|c|c|c|}
\hline \multirow{2}{*}{$v$} & \multirow{2}{*}{$n$} & \multirow{2}{*}{$c$} & \multicolumn{2}{|c|}{$U_{1}$} & \multicolumn{2}{|c|}{$U_{2}$} & \multicolumn{2}{|c|}{$U_{3}$} \\
\hline & & & $90 \%$ & $95 \%$ & $90 \%$ & $95 \%$ & $90 \%$ & $95 \%$ \\
\hline \multirow{8}{*}{1} & \multirow{3}{*}{7} & 0 & 3.141698 & 3.882563 & 3.259204 & 3.898709 & 3.018612 & 3.606341 \\
\hline & & 1 & 3.178194 & 3.943143 & 3.250642 & 3.899202 & 3.181415 & 3.910418 \\
\hline & & 2 & 3.200727 & 3.963654 & 3.221355 & 3.836194 & 3.274709 & 3.101615 \\
\hline & \multirow{5}{*}{10} & 0 & 3.142429 & 3.847422 & 3.276559 & 3.881234 & 3.475781 & 3.902103 \\
\hline & & 1 & 3.125297 & 3.882641 & 3.264698 & 3.909236 & 3.981026 & 3.154185 \\
\hline & & 2 & 3.134547 & 3.854041 & 3.264195 & 3.930944 & 3.560345 & 3.925101 \\
\hline & & 3 & 3.141698 & 3.882563 & 3.259204 & 3.898709 & 3.670165 & 3.258404 \\
\hline & & 4 & 3.178194 & 3.943143 & 3.250642 & 3.899202 & 3.413724 & 3.135169 \\
\hline \multirow{8}{*}{2} & \multirow{3}{*}{7} & 0 & 3.150345 & 3.942654 & 3.258585 & 3.893152 & 3.436196 & 3.853183 \\
\hline & & 1 & 3.184769 & 3.959394 & 3.229719 & 3.879738 & 3.066534 & 3.860473 \\
\hline & & 2 & 3.184477 & 4.013981 & 3.194513 & 3.865411 & 3.949676 & 3.866606 \\
\hline & \multirow{5}{*}{10} & 0 & 3.110997 & 3.876164 & 3.269813 & 3.845016 & 3.435706 & 3.873179 \\
\hline & & 1 & 3.097545 & 3.877075 & 3.254142 & 3.851881 & 3.846834 & 3.109103 \\
\hline & & 2 & 3.128814 & 3.897008 & 3.228095 & 3.882415 & 3.489336 & 3.951775 \\
\hline & & 3 & 3.150345 & 3.942654 & 3.258585 & 3.893152 & 3.369341 & 3.785608 \\
\hline & & 4 & 3.184769 & 3.959394 & 3.229719 & 3.879738 & 3.313953 & 3.148138 \\
\hline \multirow{8}{*}{3} & \multirow{3}{*}{7} & 0 & 3.135834 & 3.938231 & 3.231079 & 3.904221 & 3.889142 & 3.348875 \\
\hline & & 1 & 3.168948 & 3.963871 & 3.268771 & 3.900417 & 3.971983 & 3.771145 \\
\hline & & 2 & 3.211631 & 3.976917 & 3.236295 & 3.834328 & 3.097121 & 3.096853 \\
\hline & \multirow{5}{*}{10} & 0 & 3.109786 & 3.871166 & 3.275329 & 3.893187 & 3.419819 & 3.801354 \\
\hline & & 1 & 3.101788 & 3.905817 & 3.254481 & 3.940258 & 3.876775 & 3.313559 \\
\hline & & 2 & 3.134611 & 3.907431 & 3.246316 & 3.920187 & 3.421646 & 3.916297 \\
\hline & & 3 & 3.135834 & 3.938231 & 3.231079 & 3.904221 & 3.601593 & 3.065644 \\
\hline & & 4 & 3.168948 & 3.963871 & 3.268771 & 3.900417 & 3.242489 & 3.029839 \\
\hline
\end{tabular}

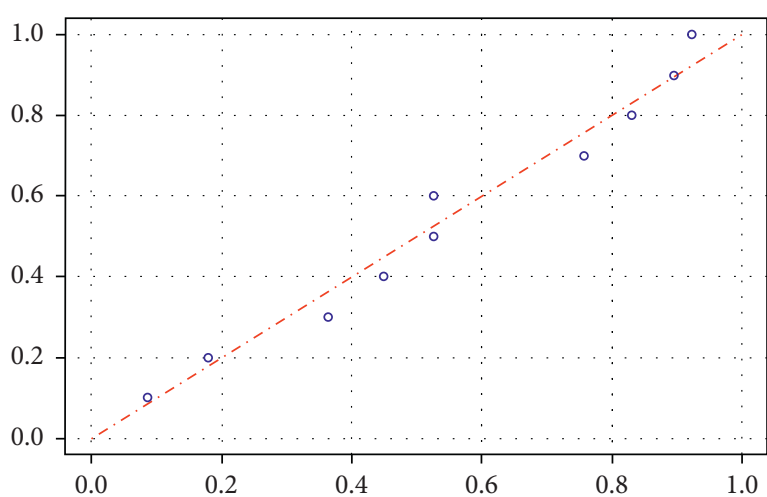

(a)

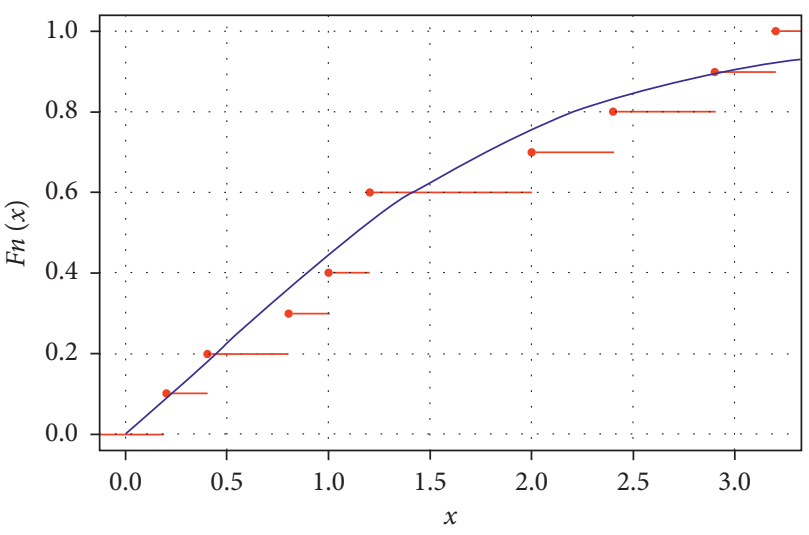

(b)

FIgURe 1: QQ plot and CDF of the real data set I on the EPL distribution. (a) QQ plot of sample vs theoretical distribution; alpha=1, beta $=1.1$, and theta $=1$. (b) CDF for sample and theoretical CDF.

distributions plot and overlay the theoretical EPL distribution, the quantile-quantile (Q-Q) plots are also presented under the maximum flood level data, as shown in Figure 2, which also imply that the EPL distribution can be used as a proper model to fit this data.

Then, by using the BLUE coefficients in Tables 1 and 2, we have

$$
\begin{aligned}
\mu^{*} & =\sum_{j=1}^{n} p_{j} X_{j: n}=0.246173, \\
\sigma^{*} & =\sum_{j=1}^{n} q_{j} X_{j: n}=0.099934 .
\end{aligned}
$$




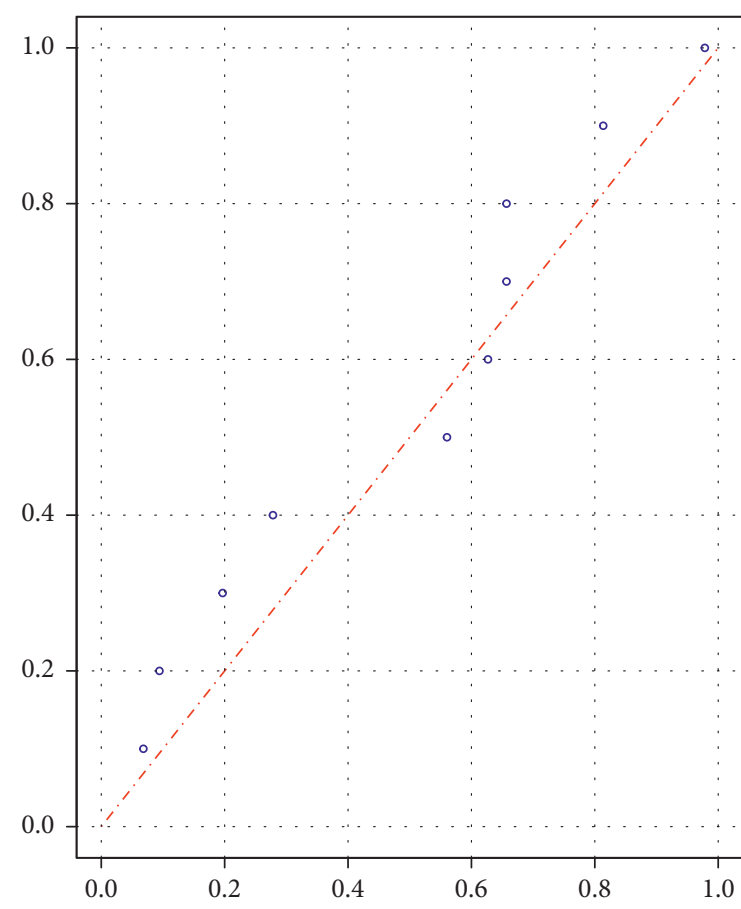

(a)

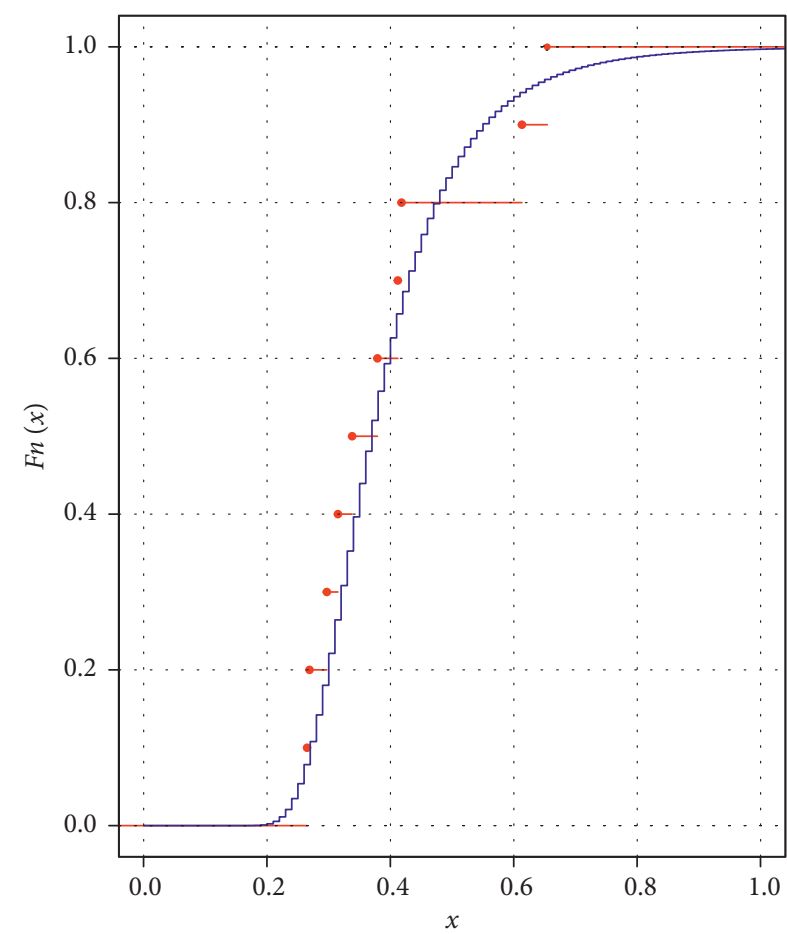

(b)

FIgURE 2: QQ plot and CDF of the real data set II on the EPL distribution.

\section{Conclusion}

In this paper, we have considered the EPL distribution when data are available in the form of order statistics. We first presented expressions for the single, double, triple, and quadruple moments. By using these moments, we have calculated the BLUEs of the location and scale parameters and the coefficient of skewness and kurtosis for some linear pivotal quantities. In the simulation study, we observed that the variance of the BLUEs increases when a high censoring level is taken into account; however, it decreases when a large value of parameter $v$ is considered corresponding to a fixed value of parameters $\delta$ and $\gamma$. Also, the covariance of the BLUEs decreases as the censoring level increases while it increases when the sample size increases and it decreases as $v$ increases. We next considered the distributions of the pivotal quantities in terms of Edgeworth approximation. These pivotal quantities are used to construct the interval estimation for the location and scale parameters. From our finding, we see that the moments of order statistics of the distribution are well behaved. This will encourage the study of the other properties of order statistics for a future research.

\section{Data Availability}

The data used to support the findings of this study are included within the article.

\section{Conflicts of Interest}

The authors declare that there are no conflicts of interest regarding the publication of this paper.

\section{Acknowledgments}

The authors extend their sincere appreciation to the Deanship of Scientific Research at King Saud University for funding this work through research group no. RG-1438-086.

\section{References}

[1] S. K. Ashour and M. A. Eltehiwy, "Exponentiated power Lindley distribution," Journal of Advanced Research, vol. 6, no. 6, pp. 895-905, 2015.

[2] S. Nadarajah, H. S. Bakouch, and R. Tahmasbi, "A generalized lindley distribution,” Sankhya B, vol. 73, no. 2, pp. 331-359, 2011.

[3] M. E. Ghitany, D. K. Al-Mutairi, N. Balakrishnan, and L. J. AlEnezi, "Power Lindley distribution and associated inference," Computational Statistics \& Data Analysis, vol. 64, pp. 20-33, 2013.

[4] V. K. Sharma, S. K. Singh, U. Singh, and F. Merovci, "The generalized inverse lindley distribution: a new inverse statistical model for the study of upside-down bathtub data," Communications in Statistics-Theory and Methods, vol. 45, no. 19, pp. 5709-5729, 2016.

[5] S. Nedjar and H. Zeghdoudi, "On Pseudo lindley distribution: properties and applications," New Trends in Mathematical Science, vol. 1, no. 5, pp. 59-65, 2017.

[6] A. Beghriche and H. Zeghdoud, "A size biased gamma lindley distribution," Thailand Statistician, vol. 17, pp. 179-189, 2019.

[7] H. A. David and H. N. Nagaraja, Order Statistics, Wiley, New York, NY, USA, 3rd edition, 2003.

[8] B. C. Arnold, N. Balakrishnan, and H. N. Nagaraja, A First Course in Order Statistics, John Wiley, New York, NY, USA, 2003. 
[9] N. Balakrishnan and A. C. Cohen, Order Statistics and Inference: Estimation Methods, Academic Press, San Diego, CA, USA, 1991.

[10] N. Balakrishnan and M. Ahsanullah, "Relations for single and product moments of record values from exponential distribution," Journal of Applied Statistical Science, vol. 2, pp. 7387, 1995.

[11] N. Balakrishnan and K. S. Sultan, "Recurrence relations and identities for moments of order statistics," in Handbook of Statistics, N. Balakrishnan and C. R. Rao, Eds., vol. 16, pp. 149-228, Elsevier, Amsterdam, Netherlands, 1998.

[12] H. J. Malik, N. Balakrishnan, and S. E. Ahmed, "Recurrence relations and identities for moments of order statistics. I: arbitrary continuous distribution," Communications in Statistics-Theory and Methods, vol. 17, pp. 2623-2655, 1988.

[13] M. A. W. Mahmoud, K. S. Sultan, and S. M. Amer, "Order statistics from inverse weibull distribution and associated inference," Computational Statistics \& Data Analysis, vol. 42, no. 1-2, pp. 149-163, 2003.

[14] A. İ. Genç, "Moments of order statistics of topp-leone distribution," Statistical Papers, vol. 53, no. 1, pp. 117-131, 2012.

[15] S. M. T. K. MirMostafaee, "On the moments of order statistics coming from the Topp-Leone distribution," Statistics \& Probability Letters, vol. 95, pp. 85-91, 2014.

[16] N. Balakrishnan, X. Zhu, and B. Al-Zahrani, "Recursive computation of the single and product moments of order statistics from the complementary exponential-geometric distribution," Journal of Statistical Computation and Simulation, vol. 85, no. 11, pp. 2187-2201, 2015.

[17] D. Kumar and S. Dey, "Power generalized Weibull distribution based on order statistics," Journal of Statistical Research, vol. 51, pp. 61-78, 2017.

[18] K. S. Sultan, A. Childs, and N. Balakrishnan, "Higher order moments of order statistics from the power function distribution and Edgeworth approximate inference," in Advances in Stochastic Simulation and Methods, N. Balakrishnan, V. B. Melas, and S. Ermakove, Eds., pp. 245-282, Birkhauser, Boston, MA, USA, 2000.

[19] K. S. Sultan and W. S. AL-Thubyani, "Higher order moments of order statistics from the Lindley distribution and associated inference," Journal of Statistical Computation and Simulation, vol. 86, no. 17, pp. 3432-3445, 2016.

[20] D. Kumar, S. Dey, and S. Nadarajah, "Extended exponential distribution based on order statistics," Communications in Statistics-Theory and Methods, vol. 46, no. 18, pp. 9166-9184, 2017.

[21] M. Ahsanullah and A. Alzaatreh, "Parameter estimation for the log-logistic distribution based on order statistics," REVSTAT Statistical Journal, vol. 16, pp. 429-443, 2018.

[22] D. Kumar and A. Goyal, "Order Statistics from the power Lindley distribution and associated inference with application," Annals of Data Science, vol. 6, no. 1, pp. 153-177, 2019.

[23] D. Kumar and A. Goyal, "Generalized lindley distribution based on order statistics and associated inference with application," Annals of Data Science, vol. 6, no. 4, pp. 707-736, 2019.

[24] D. Kumar, M. Kumar, and J. P. S. Joorel, "Estimation with modified power function distribution based on order statistics with application to evaporation data," Annals of Data Science, 2020.

[25] D. Kumar, M. Nassar, and S. Dey, "Inference for generalized inverse Lindley distribution based on generalized order statistics," Afrika Matematika, 2020.
[26] D. K. Bhaumik and R. D. Gibbons, "One-sided approximate prediction intervals for at Leastpofm observations from a gamma population at each ofrLocations," Technometrics, vol. 48, no. 1, pp. 112-119, 2006.

[27] K. Krishnamoorthy, T. Mathew, and S. Mukherjee, "Normalbased methods for a gamma distribution," Technometrics, vol. 50, no. 1, pp. 69-78, 2008.

[28] D. K. Bhaumik, K. Kapur, and R. D. Gibbons, "Testing parameters of a gamma distribution for small samples," Technometrics, vol. 51, no. 3, pp. 326-334, 2009.

[29] R. Dumonceaux and C. E. Antle, "Discrimination between the log-normal and the weibull distributions," Technometrics, vol. 15 , no. 4 , pp. 923-926, 1973. 